\title{
High strain rate in situ micropillar compression of a Zr-based metallic glass
}

\author{
Rajaprakash Ramachandramoorthy ${ }^{1,2, a)}$, Fan Yang $^{3}$, Daniele Casari ${ }^{2}$, Moritz Stolpe ${ }^{4}$, \\ Manish Jain ${ }^{2}$, Jakob Schwiedrzik ${ }^{2}$, Johann Michler ${ }^{2}$, Jamie J. Kruzic ${ }^{5}$, James P. Best ${ }^{1,5,6, a)}$ (D) \\ ${ }^{1}$ Max-Planck-Institut für Eisenforschung GmbH, Max-Planck-Straße 1, 40237 Düsseldorf, Germany \\ ${ }^{2}$ Laboratory for Mechanics of Materials and Nanostructures, Empa - Swiss Federal Institute for Materials Science and Technology, 3603 Thun, \\ Switzerland \\ ${ }^{3}$ Institut für Materialphysik im Weltraum, Deutsches Zentrum für Luft- und Raumfahrt (DLR), 51170 Köln, Germany \\ ${ }^{4}$ Heraeus Amloy Technologies GmbH, Heraeusstraße 12-14, 63405 Hanau, Germany \\ ${ }^{5}$ School of Mechanical and Manufacturing Engineering, University of New South Wales (UNSW Sydney), Sydney, NSW 2052, Australia \\ ${ }^{6}$ Institut für Metallkunde und Materialphysik, RWTH Aachen University, 52056 Aachen, Germany \\ a) Address all correspondence to these authors.e-mails: r.ram@mpie.de; j.best@mpie.de \\ Received: 17 November 2020; accepted: 24 March 2021; published online: 20 April 2021
}

High strain rate micromechanical testing can assist researchers in elucidating complex deformation mechanisms in advanced material systems. In this work, the interactions of atomic-scale chemistry and strain rate in affecting the deformation response of a Zr-based metallic glass was studied by varying the concentration of oxygen dissolved into the local structure. Compression of micropillars over six decades of strain rate uncovered a remarkable reversal of the strain rate sensitivity from negative to positive above $\sim 5 \mathrm{~s}^{-1}$ due to a delocalisation of shear transformation events within the pre-yield linear regime for both samples, while a higher oxygen content was found to generally decrease the strain rate sensitivity effect. It was also identified that the shear band propagation speed increases with the actuation speed, leading to a transition in the deformation behaviour from serrated to apparent non-serrated plastic flow at $\sim 5 \mathrm{~s}^{-1}$.

\section{Introduction}

Bulk metallic glasses (BMGs) possess an amorphous structure that exhibits superior strength compared to their crystalline counterparts. Such superior material properties make them attractive for many applications including MEMS devices, high performance gears, cutting tools, armour-piercing projectiles, spacecraft components, etc. [1-4]. Therefore, understanding the mechanical properties of BMGs at both macro- and microscales has been a topic of intense research over the last few decades [5]. At the macroscale with millimetre sized samples, several mechanical testing studies have been previously conducted under a variety of loading conditions [6]. The common conclusion from a majority of these studies is that BMGs, though exhibiting high strength, show very little plastic strain before a localised shear band-mediated failure. Previous studies that explored the rate-dependency of the BMG mechanical properties from quasi-static to $10,000 \mathrm{~s}^{-1}$ strain rates typically find a negative strain rate sensitivity (SRS) with increasing strain rate
$[7,8]$. The negative SRS of BMGs and failure via shear-banding are optimally suited for unique dynamic applications such as in kinetic energy penetrators, which require a self-sharpening mechanism [9].

At small scales, nanoindentation and micropillar compression experiments are typically the preferred mechanical testing methods. Though there exists extensive literature exploring the rate-dependency of BMGs at small scales, they are primarily limited to speeds of $300 \mathrm{mN} \mathrm{s}^{-1}$ (loading rate) and $100 \mathrm{~nm} \mathrm{~s}^{-1}$ in nanoindentation and micropillar compression, respectively $[10,11]$. These speeds translate to a maximum indentation and uniaxial micropillar compression strain rate of $\sim 1 \mathrm{~s}^{-1}[12,13]$. Within this strain rate envelope using nanoindentation testing, studies have identified three distinct regimes of BMG deformation namely, homogenous, serrated and non-serrated as a function of increasing strain rate [14]. The interpretation of such nanoindentation results, however, is not straight-forward due to the complex stress-state and evolving deformation volume 
during the indent. This makes micropillar compression with its uniform uniaxial stress state more suitable for investigating the rate-dependent behaviour of BMGs. Unfortunately, owing to the lack of specialised instrumentation the strain rates achievable under micropillar compression has been largely limited to less than $0.1 \mathrm{~s}^{-1}$. Though recently, the authors of this study reported the micromechanical properties of fused silica and 3D-printed polymer structures at strain rates up to $1000 \mathrm{~s}^{-1}$ using a piezobased testing platform $[15,16]$. There are also a small number of other recent key studies on copper micropillar compression conducted at strain rates up to $100 \mathrm{~s}^{-1}$ and nanoindentation of coarse-grained aluminium and nanocrystalline nickel performed at indentation strain rates up to $100 \mathrm{~s}^{-1}[17,18]$.

In metallic glasses, the atomic-scale ordering known as short- and medium-range order (SRO and MRO) play a determining role over their properties $[19,20]$ for both homogeneous and inhomogeneous deformation modes [21]. Variations in the local clustering of atoms lead to changes in shear band dynamics and density [22]; local soft spots and/or high medium range order in the structure act as sites with a high propensity for shear transformation zone (STZ) activation [20,23], with clear consequences on the transition to plastic flow during material deformation [24]. Changing the local chemical structure can also affect the response at high strain rates, as demonstrated by Inoue and co-workers for $\mathrm{Zr}-\mathrm{Cu}-\mathrm{Al}$ amorphous alloys with various amounts of $\mathrm{Ni}$ additions via Charpy impact testing [25], while the degree of hybridised bonding has also been shown to be linked to the fracture toughness of metallic glasses [26]. From instrumented nanoindentation experiments, Schuh and Nieh demonstrated that the composition of the glass alloy had a significant effect on the serrated flow behaviour and sensitivity to strain-rate effects, further supporting the importance of local atomic arrangements on the mechanical response [27].

Elemental contaminant species can also play a mediating role on both the local structural ordering and the mechanical response of metallic glasses. Recently, the authors of this study investigated the role of oxygen contamination on the fracture toughness of a Zr-based BMG alloy produced by laser additive manufacturing [28]. The higher oxygen containing ( $1270 \mathrm{ppm})$ laser-processed material was significantly more brittle than its as-cast counterpart, where differences were reasoned to be due to the inclusion of oxygen in the local atomic ordering. Moreover, recent work with Zr-based BMGs have shown that imbuing the glass with contaminants can have a strong effect on the undercooling ability of the glass (GFA), as oxygen in undercooled Zr-based melts induces heterogeneous nucleation [29-32]. However, there is little current understanding of the role of oxygen in affecting the STZ local chemistry and the small-scale mechanical response of metallic glasses.

Available methods to analyse the local structural order in metallic glasses include local probing of the atomic pair distribution function and characteristic MRO cluster size using nanobeam electron diffraction and variable resolution fluctuation electron microscopy using a transmission electron microscope [20, 33-35]; however, such transmission electron microscopy methods only allow for a snapshot of one very localised region of the glass. Alternatively, a more statistically averaged picture of the atomic structure can be obtained by microdiffraction using high-energy X-rays (HEXRD) to measure highly-resolved determinations of the real-space pair distribution functions using high-resolution diffraction patterns [36, 37]. In this work, the latter method was utilised and the role of oxygen on the atomic structure and mechanical response was investigated for a cast $\mathrm{Zr}$-based metallic glass (AMZ4, $\mathrm{Zr}_{59.3} \mathrm{Cu}_{28.8} \mathrm{Nb}_{1.5} \mathrm{Al}_{10.4}$-subscripts indicate at.\%). Two different concentrations of oxygen were imbued into the atomic-scale order, resulting in low-oxygen containing $(A M Z 4)_{L}$ and high-oxygen containing $(\mathrm{AMZ} 4)_{\mathrm{H}}$. Synchrotron HEXRD measurements were performed to study structural variation in real-space. Then, a newly developed high strain rate nanomechanical system was utilised to compress micropillars of both materials, from quasi-static rates up to $520 \mathrm{~s}^{-1}$, to understand the fundamental mechanistic differences in deformation based on the local chemistry. Differences observed for the samples as a function of strain-rate, and between samples, were rationalised based on the local structural order.

\section{Results and discussion}

The material used was an AMZ4 alloy $\left(\mathrm{Zr}_{59.3} \mathrm{Cu}_{28.8} \mathrm{Nb}_{1.5} \mathrm{Al}_{10.4}\right.$ subscripts indicate at.\%) suction-cast with either $\sim 170$ ppm oxygen $(\mathrm{AMZ} 4)_{\mathrm{L}}$ or $\sim 845 \mathrm{ppm}$ oxygen $(\mathrm{AMZ} 4)_{\mathrm{H}}$. The maximum casting dimension was limited by the critical casting thickness of the alloy containing the greater concentration of oxygen, and as such $1.5 \mathrm{~mm}$ rods were cast to retain fully amorphous samples. Differential scanning calorimetry (DSC) results presented in Fig. 1a,b demonstrate a typical glassy thermal response, where similarities in the curve shapes and transitions (glass, supercooled liquid and crystallisation) can be observed for both samples. Differences were observed in the crystallisation peak, however, where a much wider peak of $3.2^{\circ} \mathrm{C}$ full width at half maximum (FWHM) was observed for the (AMZ4) sample compared to $1.1^{\circ} \mathrm{C}$ FWHM for the $(\mathrm{AMZ} 4)_{\mathrm{H}}$ sample. Additionally, the crystallisation peak was observed to shift to a lower temperature for the $(\mathrm{AMZ} 4)_{\mathrm{H}}$ sample. Nonetheless, crystallisation enthalpies were found to be comparable: $13.3 \mathrm{~W} / \mathrm{g} \cdot \mathrm{K}$ and $11.2 \mathrm{~W} / \mathrm{g} \cdot \mathrm{K}$ for $(\mathrm{AMZ4})_{\mathrm{L}}$ and $(\mathrm{AMZ4})_{\mathrm{H}}$, respectively. The difference in FWHM of the crystallisation peak has been previously used to indicate an inverse measure of the crystallisation rate [38]. A higher FWHM is linked to a lower nucleation rate. Thus, the kinetic barrier for crystallisation is decreased for the 
(a)

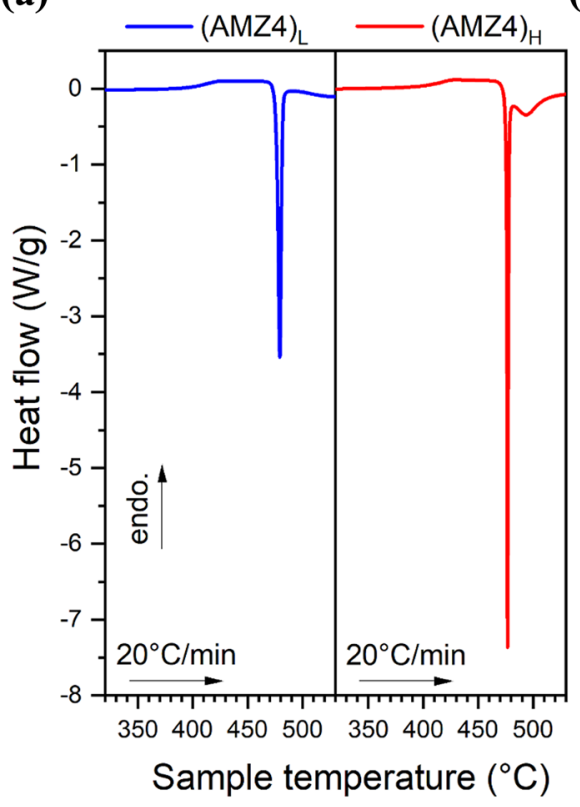

(b)

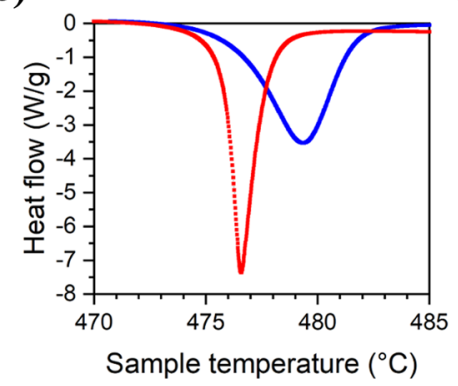

(c)

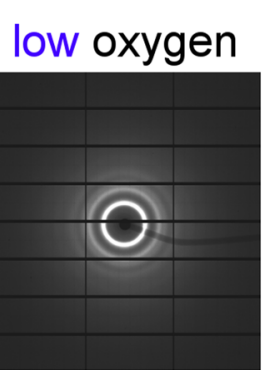

(d)

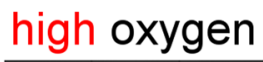

(e)

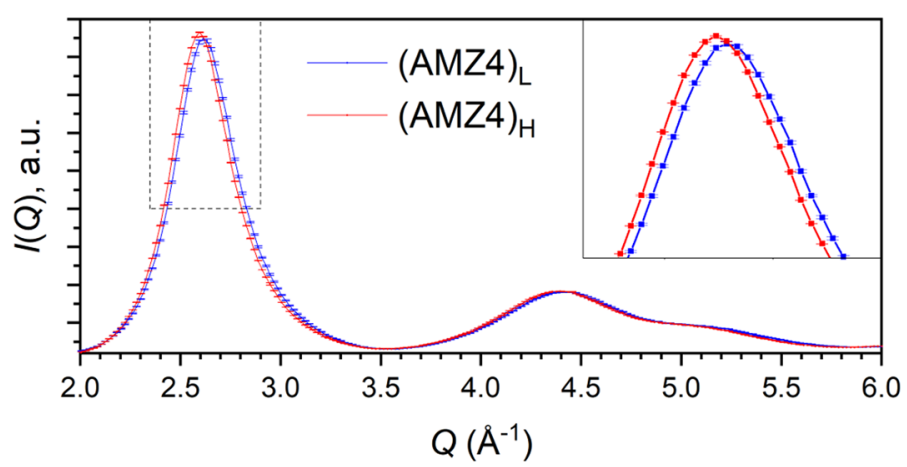

Figure 1: Characterisation of the cast AMZ4 alloys. DSC results are presented (a), together with a magnification of the crystallisation peak (b). HEXRD results from synchrotron measurements are also shown, where the diffraction patterns for both samples (c, d) were used to plot the intensity distribution $I(Q)(e)$. The grid patterns visible in the diffraction images are from the 2D detector and were masked during analysis.

$(\mathrm{AMZ} 4)_{\mathrm{H}}$ sample, which is indicative of a higher initial degree of structural organisation for the higher oxygen containing sample and is also consistent with the peak shift.

A micro-focussed high-energy X-ray beam was used to interrogate the structure of the two different $(A M Z 4)_{L}$ and $(\mathrm{AMZ} 4)_{\mathrm{H}}$ samples. HEXRD results shown in Fig. 1c,d highlight an X-ray amorphous structure with an amorphous halo visible indicating lack of long-range order. When integrating the masked diffraction data over an azimuthal range of $0^{\circ}-360^{\circ}$ to obtain the intensity signal as a function of $Q, I(Q)$, distinct and significant lateral shifts were observed in the data indicating structural variation between the amorphous alloy samples. To probe these shifts further, $I(Q)$ data for both samples were then processed using the methodology presented in Reference [39] to determine the structure function $S(Q)$ and pair distribution function (PDF, $G(r)$ ) via a Fourier transform to convert the collected diffraction data to real-space. Results of this analysis are shown in Fig. 2, where small but significant shifts are observed in both the structure function and in the real-space PDF.

Figure 2 indicates that the interatomic pair distances shift depending on the oxygen concentration dissolved into the structure. A higher oxygen concentration led to shifts of the $G(r)$ to greater $r$ values for all peaks, indicating that the interatomic distances were statistically increased due to oxygen inclusion. Magnifications of the nearest neighbour peak $\left(r_{1}\right)$ and 4 th nearest neighbour peak $\left(r_{4}\right)$ are highlighted in the figure illustrating structural changes at the length scales of SRO and MRO, respectively. It should also be noted that oxide nanocrystals are not expected to be present based on our previous TEM investigations [28]. Rather, the inclusion of oxygen is thought to cause an increased level of directional bonding in the structural ordering, as suggested in Reference [26].

To test the influence of the observed atomic-scale structural ordering differences on the mechanical response, micropillars were milled using a $\mathrm{Ga}^{+}$focussed ion beam (FIB) workstation to a nominal diameter of $\sim 3 \mu \mathrm{m}$ for testing at a range of strain rates (geometric parameters for both micro-pillar sets are provided in Supplementary Table T1). All the micromechanical tests in this study were conducted via a high stiffness piezo stack actuator with a stiffness of $\sim 1 \times 10^{7} \mathrm{~N} \mathrm{~m}^{-1}$. With respect to load-sensing, a typical strain gauge-based load cell has a stiffness of $\sim 2.38 \times 10^{5} \mathrm{~N} \mathrm{~m}^{-1}$, which limits the micromechanical testing speeds to $\sim 1 \mu \mathrm{m} \mathrm{s}^{-1}$. In this study, below this limit tests were carried out using PID-based true-displacement control using the strain gauge-based lower stiffness load cell. For testing speeds above $1 \mu \mathrm{m} \mathrm{s}^{-1}$, a piezo-based load cell with a higher stiffness of $\sim 2.56 \times 10^{7} \mathrm{~N} \mathrm{~m}^{-1}$ was used instead, allowing intrinsic displacement-controlled testing. It should be noted that for the Alemnis AG testing setup used in this study, the support hardware has a maximum frequency limit for signal output and acquisition of $\sim 1 \mathrm{MHz}$ (Supplementary Figure S1). 

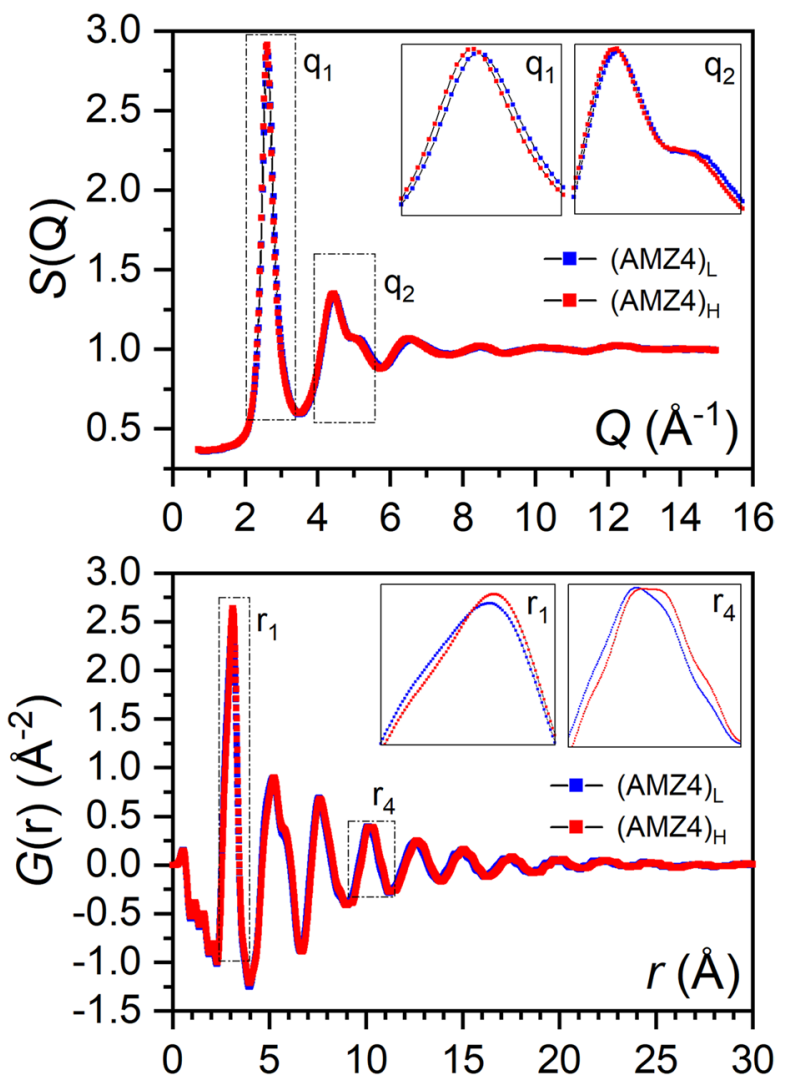

Figure 2: Synchrotron diffraction analysis of cast AMZ4. The $S(Q)$ was determined from the $I(Q)$ data where distinct lateral shifts are seen between samples, while the real-space $G(r)$ data highlights interatomic shifts between samples in both the nearest neighbour $\left(r_{1}\right)$ and 4 th nearest neighbour $\left(r_{4}\right)$ peaks (note that commensurate lateral shifts are seen for all peaks in $G(r)$ data).
It should also be noted that dynamic mechanical testing at the microscale has several benefits over macroscale testing, including higher strain rates due to smaller sample size and faster stress wave equilibration times. As shown in Fig. 3 for example, the duration of a dynamic experiment conducted at $1000 \mathrm{~s}^{-1}$ strain rate is $\sim 70 \mu \mathrm{s}$, both in the macro- and microscale samples. With a longitudinal elastic wave speed $(E / \rho)^{0.5}$ estimated to be $\sim 3560 \mathrm{~m} \mathrm{~s}^{-1}$ for an AMZ4 elastic modulus $E$ of $85 \mathrm{GPa}$ with density $\rho$ of $6.7 \mathrm{~g} \mathrm{~cm}^{-3}$, the wave travel time in a macroscale sample of $9 \mathrm{~mm}$ thickness (the typical thickness of samples used in Kolsky bar measurements is between 6 and $12 \mathrm{~mm}$ ) is $\sim 2.5 \mu \mathrm{s}$, while the wave travel time in a $5 \mu \mathrm{m}$ AMZ4 metallic glass micropillar is $\sim 1.4 \mathrm{~ns}$. At the macroscale, the time scale of experiment and the wave travel time is in the same order of magnitude, making the understanding of wave propagation phenomena vital [42]. In contrast, at the microscale the duration of the experiment far exceeds the wave travel time, essentially making the wave propagation phenomena irrelevant in these samples at strain rates less than $\sim 10^{5} \mathrm{~s}^{-1}$. This also means that the three elastic wave reverberations in the microscale specimen that are required for stress equilibration are typically achieved within the first 3 ns of the test [43].

Micropillars from both AMZ4 samples were compressed, and Fig. 4 shows the representative engineering stress-strain responses at various strain rates from $5 \times 10^{-4}$ to $520 \mathrm{~s}^{-1}$. From Fig. 4 a number of critical observations can be made. Firstly, a decrease is seen in the post-yield serrated flow behaviour for both samples with increasing strain rate. Second, with increasing strain rate both samples show an initial reduction in the yield

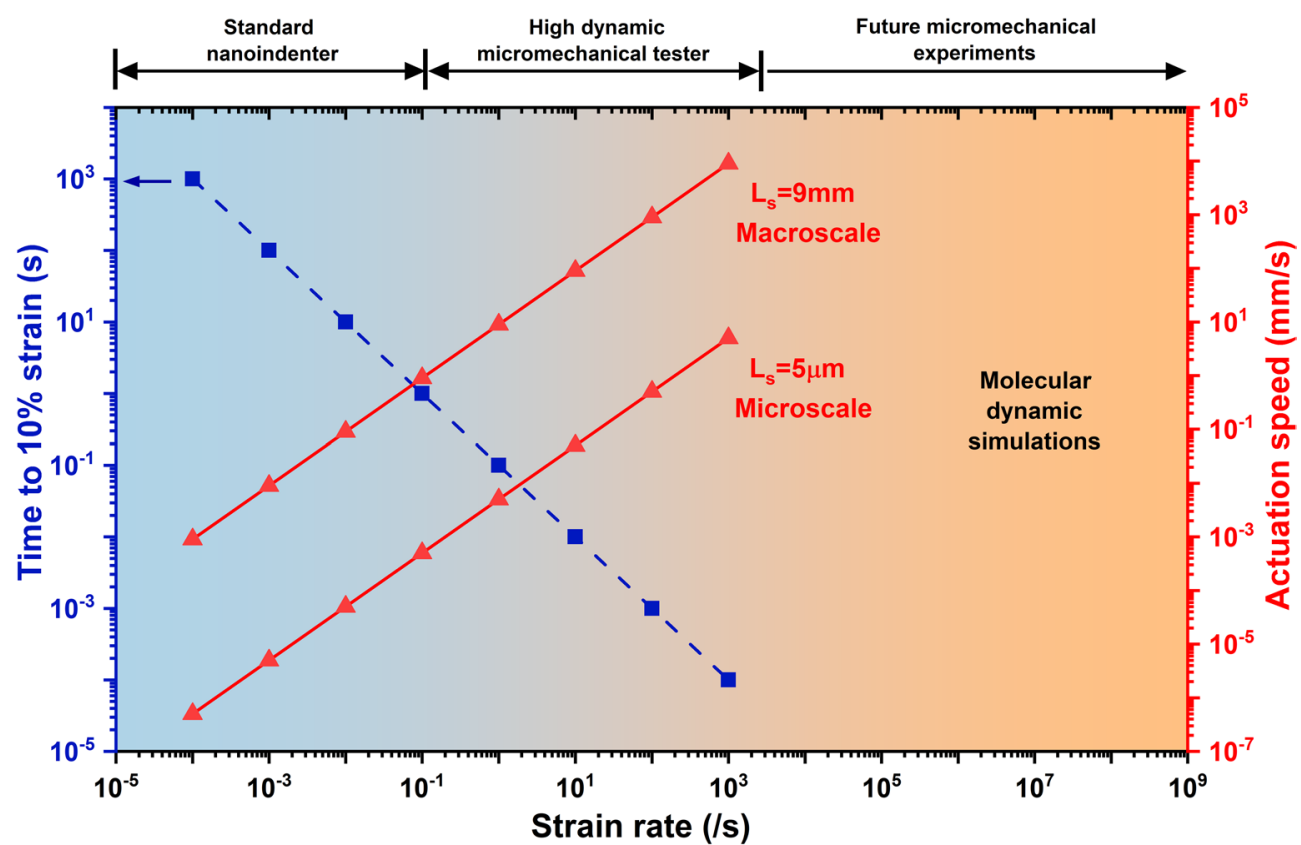

Figure 3: Comparison of testing speeds required to achieve different strain rates in experiments with microscale and macroscale samples, with a sample length $L_{s}$. The high strain rate experiments conducted in this study with the high dynamic micromechanical tester at $\sim 520 \mathrm{~s}^{-1}$ are only $3-4$ orders of magnitude lower that the strain rates typically used in molecular dynamics simulations [40, 41]. 


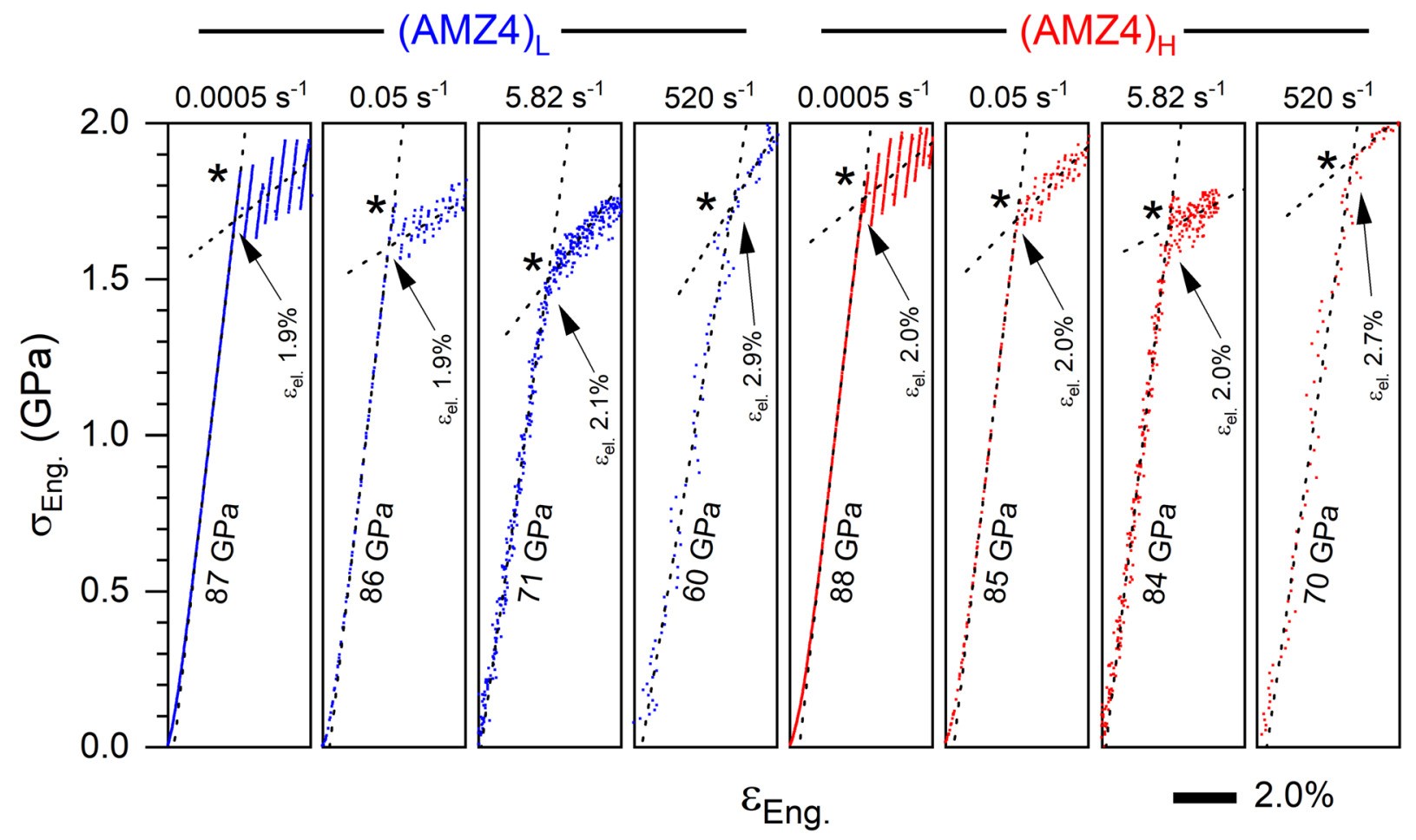

Figure 4: Representative engineering stress-strain curves for both low (blue) and high (red) oxygen concentrations in cast AMZ4. Also shown in the figure are the evaluated linear fits for the elastic slope, and the elastic strain limits for each curve based on the linear intercept fit from elastic and initial plastic components of the curve (dotted lines). The yield stress is highlighted with the * symbol. The stress-strain data for the experiments at $520 \mathrm{~s}^{-1}$ were captured at a data acquisition rate of $1 \mathrm{MHz}$ and have been moving averaged by 40 points.

strength (negative strain rate sensitivity) followed by a sharp increase at $520 \mathrm{~s}^{-1}$. Yield strength is here defined as the stress at the first deviation from linear response (i.e. stress drop at low strain rates). Finally, the apparent sensitivity of the SRS is reduced for the AMZ4 sample with an increased oxygen concentration dissolved into the structure. These observations will be discussed in more detail in the remainder of the discussion.

\section{Decreasing serration amplitudes with increasing strain rate}

AMZ4 samples exhibit a reduction in serrated flow behaviour with increasing strain rate; there is a clear transition from strong flow serration, through to moderate serration and finally little or no flow serration at $520 \mathrm{~s}^{-1}$, as seen in Fig. 4. Additionally, Fig. 5a shows in detail the post-yield data regime with connected data points where any evidence of serrated flow (discrete load drops) is minimised to the extent that it falls below the noise level. Supplementary Figure S1 and Table T2 further provide evidence that if load drops were present which overcame the noise level, the data acquisition rate was kept high enough to capture them at every strain rate reported here.

This finding is consistent with mechanistic descriptions of a number of rate-dependent studies on amorphous materials reported in literature $[6,16]$. Specifically, previous rate-dependent nanoindentation studies on BMGs also report a critical indentation strain rate between 0.1 and $10 \mathrm{~s}^{-1}$ above which load serrations apparently do not occur [27]. Similarly, in our study both $(\mathrm{AMZ4})_{\mathrm{H}}$ and $(\mathrm{AMZ4})_{\mathrm{L}}$ micropillars tested at lower strain rates of $0.0005 \mathrm{~s}^{-1}$ and $0.05 \mathrm{~s}^{-1}$ exhibit plastic flow that is largely serrated, while the pillars deformed at even higher strain rates of $5.82 \mathrm{~s}^{-1}$ and $520 \mathrm{~s}^{-1}$ show smoother stress-strain curves due to decrease in serration amplitudes to levels lower than the noise floor of the load signals. The present results on AMZ4 micropillars span approximately six orders of magnitude in strain rate and shed light upon the trends observed in prior studies of BMGs, albeit under compressive loading conditions [14, 44].

Irrespective of the oxygen content differences between $(\mathrm{AMZ} 4)_{\mathrm{L}}$ and $(\mathrm{AMZ} 4)_{\mathrm{H}}$, an analysis of the serrations revealed that the load drop speed, corresponding to the shear band propagation speed $\left(v_{\text {S.B. }}\right)$, increased from $\sim 0.02$ to $\sim 1 \mu \mathrm{m} \mathrm{s}^{-1}$ as the strain rate was increased from 0.0005 to $0.05 \mathrm{~s}^{-1}$ (Fig. 5b). The ratio between the shear band propagation speed and actuation speed $\left(v_{\mathrm{ac}}\right)$ has been previously established as the critical transition factor for BMG plastic deformation to switch from a serrated to a non-serrated regime [44]. A higher ratio leads to a serrated plastic flow and when the ratio approaches to 1 or lower, a non-serrated plastic flow results as the deformation is overwhelmed by the actuation speed. As seen from Fig. 5, as the strain rate is increased by two orders of magnitude the 
(a)
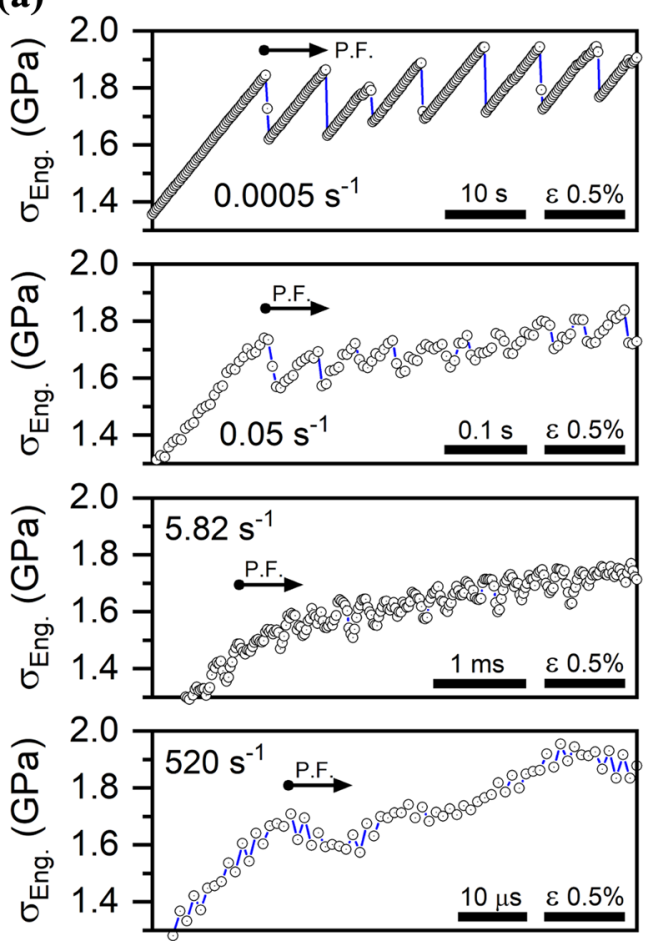

(b)

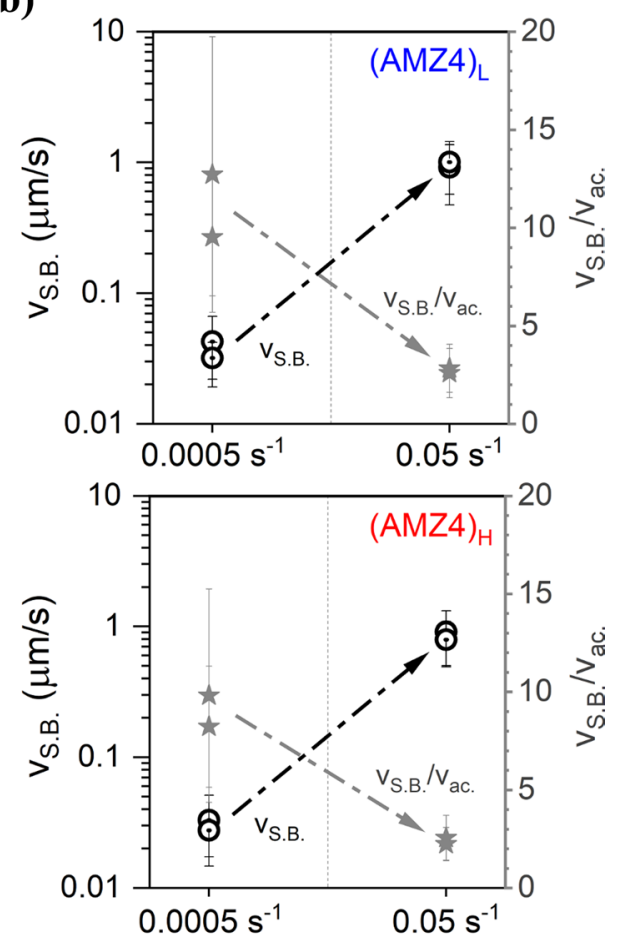

Figure 5: Engineering stress-time (unfiltered raw) data for all four actuation speeds for (AMZ4), are shown (a), with linear connections between data points (arrows in (a) indicate the start of the plastic flow (P.F.) regime). The impact of actuation speed ( $v_{\mathrm{ac}}$ ) on the shear band propagation speed $\left(v_{S . B}\right)$ in AMZ4 micropillars is highlighted in (b). The shear band propagation speed was analysed for two representative pillars tested at strain rates of $0.0005 \mathrm{~s}^{-1}$ and $0.05 \mathrm{~s}^{-1}$ (where serrations were present) for both $(A M Z 4)_{\mathrm{L}}$ and $(A M Z 4)_{\mathrm{H}}$. It should be noted that each analysis point in (b) was determined from 11 serrations. Data for the analysed serrations are provided in a supplementary dataset.

ratio between the shear band propagation speed and the actuation speed decreased from $\sim 10$ to 2 . Therefore, we posit for both $(\mathrm{AMZ} 4)_{\mathrm{L}}$ and $(\mathrm{AMZ4})_{\mathrm{H}}$ samples that the transition strain rate is around $5 \mathrm{~s}^{-1}$, and therefore seems to be insensitive to the oxygen content in the samples. The sharp transition in strength values, seen in Fig. 4 at strain rates beyond $5 \mathrm{~s}^{-1}$ in both $(\mathrm{AMZ4})_{\mathrm{L}}$ and $(\mathrm{AMZ} 4)_{\mathrm{H}}$ samples, provide support to this hypothesis.

It should be noted that the strain gauge-based load cell is capable of capturing the load drops or shear band propagations during low strain rate experiments (up to $\sim 0.05 \mathrm{~s}^{-1}$ ), as the elastic energy release during the drops is not large enough to excite the resonance frequencies of the load cell and the speeds of the load drops are still slow enough at $<1 \mu \mathrm{m} \mathrm{s}^{-1}$ (Fig. 5b). Further, the shear band propagations are intrinsically linked to the ratio between elastic strain energy release and the dissipated plastic energy. In the current study, the BMG micropillar sample has a stiffness of $\sim 8.1 \times 10^{4} \mathrm{~N}$ $\mathrm{m}^{-1}$ and the device stiffness with the strain gauge-based load cell (used for tests at lower strain rates) is $\sim 2.38 \times 10^{5} \mathrm{~N} \mathrm{~m}^{-1}$ and the piezo-electric load cell is $\sim 2.56 \times 10^{7} \mathrm{~N} \mathrm{~m}^{-1}$. As such, in either of the device configurations we have met the criteria for stable shear band propagation where the machine stiffness should be higher than the sample stiffness [45]. Additionally, the current authors have previously shown that both the strain gauge-based and the piezo-electric load cell result in nominally equivalent stress-drop or shear band propagation speeds in quasi-brittle amorphous silica at a strain rate of $\sim 0.1 \mathrm{~s}^{-1}$ [16]. In the present study at lower strain rates, therefore, each stress-drop can be correlated to the nucleation and propagation of an individual stable shear band, in line with literature [44].

Further, thermal modelling using the finite element method (FEM) was carried out to investigate the potential influence of bulk heating on the mechanical behaviour of AMZ4 micropillars at high strain rates. While the boundary condition on the top of the pillar with the diamond flat-punch has a clear effect on the temperature distribution and maximum temperature achieved in the pillar during the test, the maximum temperature rise even with a purely insulating boundary was only moderate at below $\sim 2 \mathrm{~K}$. This negligible increase in temperature due to bulk heating in the micropillars at high strain rates of $\sim 500 \mathrm{~s}^{-1}$ is unlikely to influence the mechanical behaviour of AMZ4.

\section{Transition from a negative to positive SRS}

A negative SRS is common for MGs at high temperatures and low strain rates, where typically the deformation is serrated [46-49]. As highlighted in Fig. 6, both AMZ4 samples also exhibit a moderate 
(a)

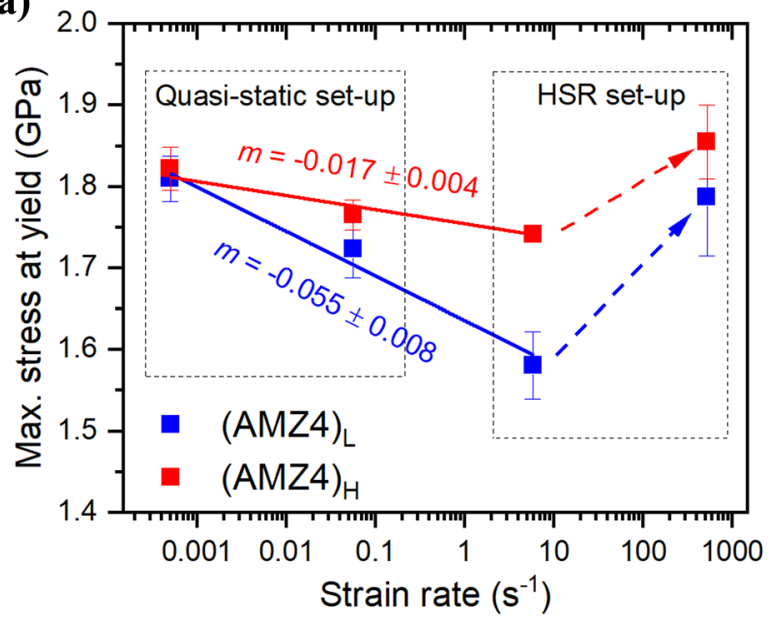

(b)

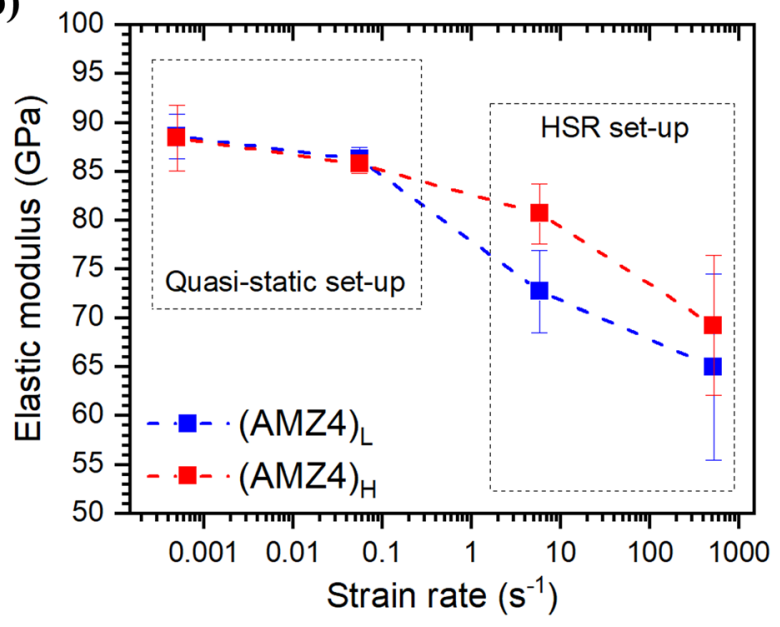

Figure 6: Results for the evaluated yield stress based on the maximum stress at yield (a), and the apparent elastic modulus from micro-pillar compression tests (b). Regions of negative strain rate sensitivity are fit with a linear function (solid lines), while remaining dotted lines are used to guide the eye. Error bars represent one standard deviation for (a-b).

negative SRS between strain rates of $0.0005 \mathrm{~s}^{-1}$ and $5.82 \mathrm{~s}^{-1}$. A quantification of the SRS $(m=d \ln \sigma / d \ln \varepsilon)$ leads to values of $-0.055 \pm 0.008$ for $(\mathrm{AMZ} 4)_{\mathrm{L}}$ and $-0.017 \pm 0.004$ for $(\mathrm{AMZ} 4)_{\mathrm{H}}$ when considering the maximum stress at yield. Beyond $5.82 \mathrm{~s}^{-1}$, a clear transition from negative to positive SRS is seen for both $(\mathrm{AMZ4})_{\mathrm{H}}$ and $(\mathrm{AMZ4})_{\mathrm{L}}$ samples. This severe change in rate-sensitivity again supports a change in deformation mechanism at strain rates beyond $5.82 \mathrm{~s}^{-1}$, with implications for the use of BMGs for self-sharpening projectiles where a negative strain rate sensitivity is preferred. Similarly, previous dynamic testing studies on amorphous silica micropillars also showed an increase in SRS at strain rates beyond $\sim 6 \mathrm{~s}^{-1}[16]$. Interestingly, Dubach et al. identified a similar transition from negative to positive SRS in a Zr-based BMG as the temperature was decreased from 300 to $77 \mathrm{~K}$ during constant strain rate tests and hypothesised that a similar transition in SRS could also be achieved by increasing the strain rates at aiven temperature [50]. This is because shear banding based deformation is a thermally activated process, which depends on the testing temperature or in turn the testing strain rate. High strain rate testing can be thought of as being synonymous to decreasing the testing temperature, as the system inherently has a much-reduced time for the thermal fluctuations to influence the mechanical behaviour. Conventional macroscale high strain rate tests using Split-Hopkinson pressure bar methods for Zr-based BMGs have shown a relative strain rate insensitivity for the peak stress at room temperature above $1000 \mathrm{~s}^{-1}$, however bulk-scale tests may miss sensitive transitions in material deformation at strain rates between 0.1 and $1000 \mathrm{~s}^{-1}[7,8]$.

From Fig. 6, it can also be seen that at $520 \mathrm{~s}^{-1}$ a decrease in the elastic slope is observed for both samples, along with increases in the elastic strain limit (See Fig. 4) and yield strength. As such, it is postulated that at higher strain rates, there is increasing influence of STZ activation within the elastic strain limit for both (AMZ4) and $(\mathrm{AMZ} 4)_{\mathrm{H}}$, suggesting a delocalisation of activated STZs. It should be noted that the elastic-loading in micropillar compression can be affected by the frame stiffness and taper angle (see Supplementary Table T1), and that micropillar compression is not an ideal approach for accurate quantification of the elastic modulus. However, in this study the focus is on observed trends rather than absolute values. Additionally, between the low and high strain rate setups, the device stiffness does change from $\sim 2.38 \times 10^{5}$ to $\sim 2.56 \times 10^{7} \mathrm{~N} \mathrm{~m}^{-1}$ (due to the replacement of strain gauge-based load cell with piezoelectric load cell at high strain rates) and the displacements are appropriately corrected for this stiffness change during analysis. Further, it was found that for the $(\mathrm{AMZ4})_{\mathrm{H}}$ case, the modulus and elastic strain limit identified at $5.82 \mathrm{~s}^{-1}$ using the high strain rate setup was similar to those obtained at $0.0005 \mathrm{~s}^{-1}$ and $0.05 \mathrm{~s}^{-1}$ using the low strain rate setup. This result further justifies the comparison of modulus calculated from experiments conducted using the low and high strain rate setups.

SEM imaging analysis of the compressed micro-pillars (Fig. 7) show comparable shear band patterns between the $0.0005 \mathrm{~s}^{-1}$ and $520 \mathrm{~s}^{-1}$ tests (6 decades of strain rate), indicating that both samples deform in the plastic regime via inhomogeneous shear localisation (i.e. through the percolation of STZs and propagation of shearbands). Thus, at high strain rates, even though shear band propagations are present, the stress-strain signatures are non-serrated simply because the shear band propagation speed is overwhelmed by the cross-head or actuation speed, and the signature of the load drop is overshadowed by the travel of the applied cross-head. This has also been reported in several previous studies $[44,51,52]$. Differences in the stress-strain behaviour before plastic flow may, however, indicate that the initial deformation process is significantly more homogeneous through delocalised STZ activation at 


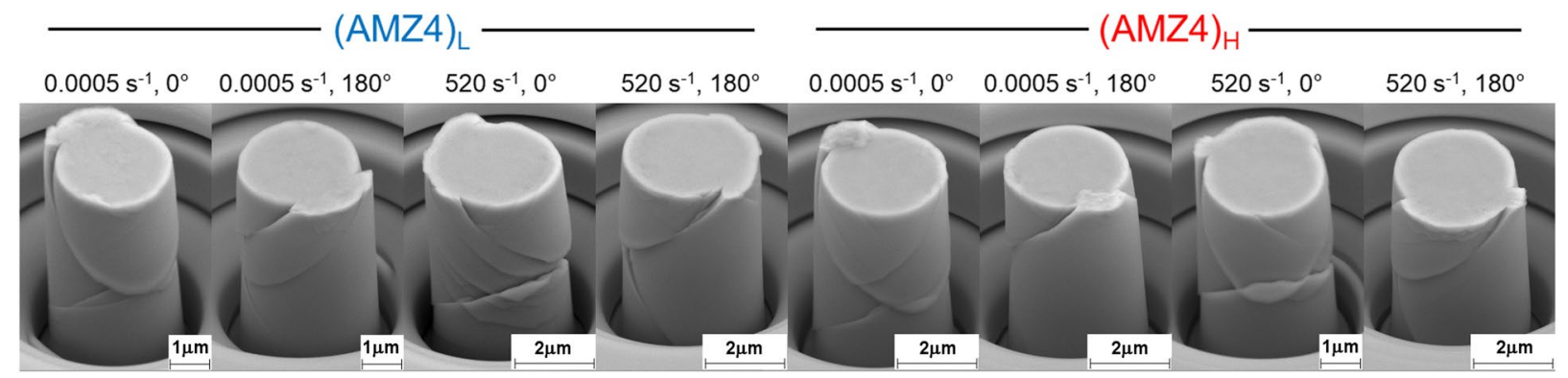

Figure 7: SEM images of post-compressed micro-pillars for both $(A M Z 4)_{L}$ and $(A M Z 4)_{H}$ at both the lowest $\left(0.0005 \mathrm{~s}^{-1}\right)$ and highest $\left(520 \mathrm{~s}^{-1}\right)$ strain-rates studied here. All pillars were compressed to a final strain of approximately $9 \%$, and all images were taken at a $45^{\circ}$ tilting angle at 2 distinct rotation angles.

higher strain rates. This delocalisation of initial STZ events leads to a delay in the formation of a primary shear band encompassing the pillar diameter, along with possible interaction between embryonic shear bands formed in the initial moments of deformation. Such a delay in the formation of the first shear band, which typically corresponds to the first plastic event, could also explain the increase in the elastic strain limit at high strain rates of $\sim 520 \mathrm{~s}^{-1}$ as seen in Fig. 4 , as a higher strain level (and consequently a higher stress level) is reached before plastic deformation is initiated. A phenomenological explanation could be that at high strain rates, though activated STZs are homogeneously distributed in the micropillar leading to a decrease in elastic modulus, the ability of the atoms in the STZs to relax to a lower energy state is severely constrained due to the microsecond timescale of the experiment [50]. This leads to a lowered resistance for the percolation of these delocalised STZs to form simultaneous multiple shear band nuclei in the micropillar $[11,12]$. Upon further application of strain, some of these shear band nuclei can propagate, leading to the formation of micropillar surface steps. Consequently, through the lower STZs percolation resistance at high strain rates there is a significant decrease in the stress-drop amplitude during shear band propagations resulting in a smoother plastic regime [50]. Previously, several nanoindentation based experimental studies and kinetic Monte Carlo based simulations on BMGs have reported that at high enough strain rates the non-serrated plastic deformation could be due to the accommodation of plastic strain by the simultaneous nucleation of shear band nuclei $[11,12,53,54]$. But as previously mentioned, the pillars show similar surface step patterns, which are manifestations of shear band propagation, across all strain rates. Henceforth, we can hypothesise that at strain rates below $\sim 5.82 \mathrm{~s}^{-1}$ the deformation of AMZ4 samples is dictated only by shear band propagation kinetics, while at higher strain rates the deformation process is possibly controlled via a combination of both shear band nucleation and propagation kinetics. In support of this hypothesis, the study of Dubach et al. described above for testing at both room and cryogenic temperatures identified that irrespective of the temperature, compressed pillars developed surface steps due to shear band propagations. The stress-strain signatures, however, were only serrated for the room temperature case while for the cryogenically tested samples the signatures showed an increased yield strength, increased elastic limit and a smooth/non-serrated plastic regime, in line with the results obtained in this study [50]. Future micropillar experiments with repeated high-speed loading and unloading segments within the elastic strain limit are required to account for potential changes in modulus and any irreversible changes in the pillar shape, in order to confirm the strain rate induced plastic softening in the apparent elastic regime. Additionally, fluctuation electron microscopy on deformed micropillars could be used to potentially quantify the density of shear band nuclei and fully propagated shear bands [55], to confirm the above-hypothesised rate-dependent deformation mechanisms.

Finally, it is noted that for (AMZ4) $)_{\mathrm{L}}$ the behaviour at high strain rates is similar to cast AMZ4 micro-pillars $(168 \pm 61 \mathrm{ppm}$ oxygen) with a smaller diameter of $\sim 2 \mu \mathrm{m}$ studied in Reference [56], where low elastic moduli values were determined (ca. 60 $\mathrm{GPa}$ ) along with increased elastic strain limits to $3 \%$. In that work, the authors argued that the decreased elastic modulus under compression was linked to the activation of STZs below the elastic limit, which could be linked to the observation of prominent load drops; observations recently supported by molecular dynamics simulations $[57,58]$. In this work, no discrete load drops were observed below the elastic limit; however, the current micro-pillars have a larger $\sim 3 \mu \mathrm{m}$ diameter which would be expected to suppress the relative influence of discrete STZs formed at local soft spots. Additionally, the samples in Reference [56] were fabricated through casting larger $42.2 \times 3.0 \times 12.9 \mathrm{~mm}^{3}$ beams rather than the $1.5 \mathrm{~mm}$ diameter rods studied here; therefore, the thermal history was also not consistent between both materials.

\section{Reduced SRS magnitude with increasing oxygen concentration}

When considering the results illustrated in Fig. 6, an initial negative SRS was observed for the (AMZ4) $)_{\mathrm{L}}$ sample ( $m$ $-0.055 \pm 0.008)$, while the $(\mathrm{AMZ} 4)_{\mathrm{H}}$ sample was three-times 
less sensitive to strain rate until $10 \mathrm{~s}^{-1}(m-0.017 \pm 0.004)$, based on evaluations of the yield stress. In a recent report, a larger negative SRS was suggested to correlate to a larger STZ volume [46]; however, in this case there may also be an influence of the directional oxygen bonding within the atomic-scale ordering which could decrease the 'BMG-like' SRS of the $(A M Z 4)_{H}$ sample. Indeed, dissolved oxygen has previously been reported to affect the intrinsic mechanical response of the AMZ4 alloy by suppressing shear transformations that occur at relatively low stress levels for cast AMZ4 [28, 56], which was attributed to the additional oxygen eliminating local atomic arrangements that have capacity for easy deformation. The HEXRD results from Fig. 2 show that changes are observed in the local ordering as the oxygen concentration is increased and suggest that the oxygen may have an affinity for $\mathrm{Zr}$ and stretch the $\mathrm{Zr}-\mathrm{Zr}$ atomic pairs. Oxygen atoms situated between $\mathrm{Zr}-\mathrm{Zr}$ pairs may also induce increased bond hybridisation which is thought to negatively affect fracture toughness of BMGs [26]. Moreover, the reduction of 'BMG-like' mechanical behaviour is supported by the DSC results of Fig. 1 where a much sharper crystallisation peak was observed (together with a slightly reduced crystallisation enthalpy) for the $(\mathrm{AMZ} 4)_{\mathrm{H}}$ sample, suggesting a reduced energetic barrier for phase transformation and structural ordering. As such, a reduction in metallic glass-like deformation for the higher oxygen containing glass reduces its sensitivity to thermally activated processes, and hence shows a reduced SRS.

\section{Conclusions}

Here we present the first insights into high strain rate micropillar compression of a $\mathrm{ZrCuAlNb}$ (AMZ4) metallic glass over a strain rate range of $5 \times 10^{-4}$ to $520 \mathrm{~s}^{-1}$. These results utilise novel nanomechanical testing infrastructure, and are the first reports of ultra-high strain rate compression on BMGs down to this size regime. Results for the yield stress show a transition at approximately $5 \mathrm{~s}^{-1}$, above which the strain rate sensitivity reverses from negative to positive with a marked increase in yield stress. This transition must be investigated at intermediate strain rates in further studies. Additionally, a reduction in the elastic modulus and the serrated flow type behaviour was observed with increasing strain-rate, with serrated flow being completely eliminated at $\sim 5 \mathrm{~s}^{-1}$. The hypothesised reason for such changes were a transition from inhomogeneous to homogeneous nucleation of STZs within the elastic regime, leading finally to an increased elastic strain limit due to the delocalised STZ activation. Interestingly, SEM imaging of the final deformed micro-pillars showed that despite the disparities in deformation response to yield, the postyield deformation still occurred through shear band formation and sliding. Finally, the sensitivity of the technique to varying materials systems is demonstrated through the study of two cast AMZ4 samples with varying concentrations of oxygen dissolved into the atomic-scale order. AMZ4 samples with higher oxygen concentrations were less sensitive to strain rate dependent effects, highlighting the role of the STZ bonding chemistry and characteristics on the shear transformation responses.

\section{Experimental materials and methods}

\section{Sample preparation}

Ingots of the alloy were prepared in a customised arc-melter from industrial high purity raw elements in a Ti-gettered high purity argon atmosphere to form the $(\mathrm{AMZ} 4)_{\mathrm{L}}$ sample. The raw elements were alloyed and the ingot was homogenised by several remelting steps. Rods were produced by melting the homogenised ingots and suction casting the melt into water-cooled copper moulds in a customised suction cast device. The $(\mathrm{AMZ} 4)_{\mathrm{H}}$ sample was prepared by mixing the high-purity ingots with a calculated wt.\% of laser-processed AMZ4 material containing a higher oxygen concentration ( $1271 \pm 41 \mathrm{ppm})$ before melting. The oxygen concentrations of the samples were analysed using ICP-OES (inductively coupled plasma optical emission spectrometry) according to DIN EN ISO 4491-4. Additional details on material preparation can be found in Reference [28].

\section{Thermal and structural analysis}

The thermal response of cast AMZ4 samples was investigated using differential scanning calorimetry (DSC, PerkinElmer DSC8000). Samples (ca. $20 \mathrm{mg}$ ) were cut using a low-speed diamond saw, cleaned in alcohol, and heated at $20{ }^{\circ} \mathrm{C} \mathrm{min}-1$ through to crystallisation. Repeat scans for the crystallised glass were then used as a corrective baseline for the glass measurements. Analysis of the local structural order for cast AMZ4 samples was achieved using high-energy X-ray diffraction measurements at the 2nd experimental hutch of the PETRA III P07 beamline at the Deutsches Elektronen-Synchrotron (DESY) in Hamburg, Germany. Three repeat measurements were taken for the cast samples with $\sim 170$ and $\sim 845$ ppm oxygen, using a microfocussed beam with width $30 \mu \mathrm{m}$ and height $2 \mu \mathrm{m}$. Relevant information regarding experimental methodology and data processing can be found in Reference [39].

\section{Micromechanical testing}

Micropillars were fabricated using a FEI Helios Nanolab 600i with consistent $\mathrm{Ga}$-ion probe currents for all pillars, resulting in negligible geometric variation (see Supplementary Table T1). Micropillar compression was performed using an Alemnis micromechanical tester with a standard strain gauge-based load cell for quasi-static testing $\left(5 \times 10^{-4}\right.$ and $\left.5 \times 10^{-2} \mathrm{~s}^{-1}\right)$, while the Alemnis high strain rate module with a piezoelectric load cell was used for the high strain rate testing $\left(5.82\right.$ and $\left.520 \mathrm{~s}^{-1}\right)$. A 
$5 \mu \mathrm{m}$ diameter diamond flat punch was used for all tests (Synton-MDP AG, Switzerland). Mechanical tests were performed within a Philips XL30 SEM, allowing for precise pillar-punch alignment and video capture during deformation (see supplementary videos). Post-deformation secondary electron images were taken using a Zeiss Auriga SEM operating at $10 \mathrm{kV}$.

\section{Thermal simulations}

Axisymmetric thermal finite element simulations were performed using Comsol Multiphysics 5.5. The micropillar was modelled standing on an AMZ4 substrate with $15 \mu \mathrm{m}$ radius and height. The boundaries of the simulated substrate to the infinite half-space below were modelled as constant temperature boundaries, e.g. it was assumed and verified that they are sufficiently far away from the pillar not to affect the solution. A mesh was generated consisting of 3,121 triangular domain elements and 173 boundary elements. The effects of radiative heat loss on the boundaries exposed to vacuum were not considered, they were modelled as isolated boundaries. For the boundary at the top of the pillar in contact with the indenter two different cases were modelled: An isolated boundary to model a high interfacial thermal resistance between indenter and micropillar and a constant temperature boundary assuming that the indenter acts as a heat sink and the interfacial thermal resistance is negligible. For the highest experimental strain rates, volumetric heating of the pillar through dissipation due to bulk plastic deformation was considered. For this, the plastic energy was determined by integrating the area under the experimental stress-strain curve and subtracting the elastic contribution and divided by the length of the experiment. This power was then introduced into the whole pillar as a body heat source. Thermal simulations were run for the full duration of the experiment $(200 \mu \mathrm{s})$ and the maximum temperature rise in the pillar was calculated.

\section{Acknowledgments}

The authors acknowledge funding from Universities Australia and the Deutsche Akademischer Austauschdienst (DAAD) through the Australia-Germany Joint Research Cooperation Scheme (UA-DAAD, Nos. 57386795 and 57447466). R.R. would like to acknowledge the EMPAPOSTDOCS-II programme, which has received funding from the European Union's Horizon 2020 Research and Innovation Programme under the Marie Skłodowska-Curie grant agreement number 754364. J.J.K. also acknowledges financial support from Australian Research Council Discovery Grant DP180101393 and the Alexander von Humboldt Foundation Friedrich Wilhelm Bessel Award. We acknowledge DESY (Hamburg, Germany), a member of the Helmholtz Association HGF, for the provision of experimental facilities. Parts of this research were carried out at PETRA III at the P07 beamline, with the support of Ann-Christin Dippel and Olof Gutowski. Finally, Leon Christiansen (MPIE) is thanked for assistance with SEM imaging of the post-compressed pillars.

\section{Funding}

Open Access funding enabled and organized by Projekt DEAL.

\section{Data availability}

The datasets generated during and/or analysed during the current study are available from the corresponding authors on reasonable request.

\section{Declarations}

Conflict of interest On behalf of all authors, the corresponding authors state that there is no conflict of interest.

\section{Open Access}

This article is licensed under a Creative Commons Attribution 4.0 International License, which permits use, sharing, adaptation, distribution and reproduction in any medium or format, as long as you give appropriate credit to the original author(s) and the source, provide a link to the Creative Commons licence, and indicate if changes were made. The images or other third party material in this article are included in the article's Creative Commons licence, unless indicated otherwise in a credit line to the material. If material is not included in the article's Creative Commons licence and your intended use is not permitted by statutory regulation or exceeds the permitted use, you will need to obtain permission directly from the copyright holder. To view a copy of this licence, visit http://creativecommons.org/ licenses/by/4.0/.

\section{Supplementary Information}

The online version contains supplementary material available at https://doi.org/10.1557/s43578-021-00187-5.

\section{References}

1. J. Schroers, Q. Pham, A. Desai, Thermoplastic forming of bulk metallic glass-a technology for MEMS and microstructure fabrication. J. Microelectromechanical Syst. 16, 240-247 (2007)

2. P. Bordeenithikasem, S.N. Roberts, D.C. Hofmann, J.M. Ratliff, B.R. Greene, J.B. Bacon, S. Sohn, J. Schroers, Measuring demisability of bulk metallic glasses for potential satellite applications through ablation experiments. Adv. Eng. Mater. 22, 2000708 (2020)

3. D.C. Hofmann, P. Bordeenithikasem, L.P. Tosi, M. Hendry, C. Yahnker, C. Sunday, A. Pate, S. Firdosy, J.J. Iten, J. Nuechterlein, 
M. Stolpe, Towards additively manufacturing excavating tools for future robotic space exploration. Eng. Rep. 2, e12219 (2020)

4. D.C. Hofmann, L.M. Andersen, J. Kolodziejska, S.N. Roberts, J.-P. Borgonia, W.L. Johnson, K.S. Vecchio, A. Kennett, Optimizing bulk metallic glasses for robust, highly wear-resistant gears. Adv. Eng. Mater. 19, 1600541 (2017)

5. J.J. Kruzic, Bulk metallic glasses as structural materials: a review. Adv. Eng. Mater. 18, 1308-1331 (2016)

6. C.A. Schuh, T.C. Hufnagel, U. Ramamurty, Mechanical behavior of amorphous alloys. Acta Mater. 55, 4067-4109 (2007)

7. W. Zheng, Y.J. Huang, G.Y. Wang, P.K. Liaw, J. Shen, Influence of strain rate on compressive deformation behavior of a $\mathrm{Zr}-\mathrm{Cu}-\mathrm{Ni}$ Al bulk metallic glass at room temperature. Metall. Mater. Trans. A. 42, 1491-1498 (2011)

8. W.-D. Liu, K.-X. Liu, X.-X. Xia, W.-H. Wang, The failure stress of bulk metallic glasses under very high strain rate. J. Mater. Res. 25, 1230-1234 (2010)

9. Y.M. Wang, A.M. Hodge, P.M. Bythrow, T.W. Barbee, A.V. Hamza, Negative strain rate sensitivity in ultrahigh-strength nanocrystalline tantalum. Appl. Phys. Lett. 89, 81903 (2006)

10. C.A. Schuh, T.G. Nieh, Y. Kawamura, Rate dependence of serrated flow during nanoindentation of a bulk metallic glass. J. Mater. Res. 17, 1651-1654 (2002)

11. C.A. Schuh, A.C. Lund, T.G. Nieh, New regime of homogeneous flow in the deformation map of metallic glasses: elevated temperature nanoindentation experiments and mechanistic modeling. Acta Mater. 52, 5879-5891 (2004)

12. T.G. Nieh, C. Schuh, J. Wadsworth, Y. Li, Strain rate-dependent deformation in bulk metallic glasses. Intermetallics 10, 1177$1182(2002)$

13. D. Jang, C.T. Gross, J.R. Greer, Effects of size on the strength and deformation mechanism in Zr-based metallic glasses. Int. J. Plast. 27, 858-867 (2011)

14. R. Maaß, Beyond serrated flow in bulk metallic glasses: what comes next? Metall. Mater. Trans. A. 51, 5597-5605 (2020)

15. N. Rohbeck, R. Ramachandramoorthy, D. Casari, P. Schürch, T.E.J. Edwards, L. Schilinsky, L. Philippe, J. Schwiedrzik, J. Michler, Effect of high strain rates and temperature on the micromechanical properties of 3D-printed polymer structures made by two-photon lithography. Mater. Des. 195, 108977 (2020)

16. R. Ramachandramoorthy, J. Schwiedrzik, L. Petho, C. GuerraNuñez, D. Frey, J.-M. Breguet, J. Michler, Dynamic plasticity and failure of microscale glass: rate-dependent ductile-brittle-ductile transition. Nano Lett. 19, 2350-2359 (2019)

17. B. Merle, W.H. Higgins, G.M. Pharr, Extending the range of constant strain rate nanoindentation testing. J. Mater. Res. 35, 343-352 (2020)

18. S. Breumier, S. Sao-Joao, A. Villani, M. Lévesque, G. Kermouche, High strain rate micro-compression for crystal plasticity constitutive law parameters identification. Mater. Des. 193, 108789 (2020)
19. A.L. Greer, Y.Q. Cheng, E. Ma, Shear bands in metallic glasses. Mater. Sci. Eng. R 74, 71-132 (2013)

20. K. Nomoto, A.V. Ceguerra, C. Gammer, B.S. Li, H. Bilal, A. Hohenwarter, B. Gludovatz, J. Eckert, S.P. Ringer, J.J. Kruzic, Medium-range order dictates local hardness in bulk metallic glasses. Mater. Today, in press (2021). https://doi.org/10.1016/j. mattod.2020.10.032

21. C. Suryanarayana, A. Inoue, Mechanical behavior, in Bulk Metallic Glasses. (CRC Press, 2010)

22. Z.-Y. Yang, Y.-J. Wang, L.-H. Dai, Susceptibility of shear banding to chemical short-range order in metallic glasses. Scr. Mater. 162, 141-145 (2019)

23. X. Bian, D. Şopu, G. Wang, B. Sun, J. Bednarčik, C. Gammer, Q. Zhai, J. Eckert, Signature of local stress states in the deformation behavior of metallic glasses. NPG Asia Mater. 12, 59 (2020)

24. A.S. Argon, Plastic deformation in metallic glasses. Acta Metall. 27, 47-58 (1979)

25. Y. Yokoyama, A. Kobayashi, K. Fukaura, A. Inoue, Oxygen embrittlement and effect of the addition of Ni element in a bulk amorphous $\mathrm{Zr}-\mathrm{Cu}-\mathrm{Al}$ alloy. Mater. Trans. 43, 571-574 (2002)

26. S. Evertz, V. Schnabel, M. Köhler, I. Kirchlechner, P. Kontis, Y.-T. Chen, R. Soler, B.N. Jaya, C. Kirchlechner, D. Music, B. Gault, J.M. Schneider, D. Raabe, G. Dehm, Review on quantum mechanically guided design of ultra-strong metallic glasses. Front. Mater. 7, 89 (2020)

27. C.A. Schuh, T.G. Nieh, A nanoindentation study of serrated flow in bulk metallic glasses. Acta Mater. 51, 87-99 (2003)

28. J.P. Best, H.E. Ostergaard, B. Li, M. Stolpe, F. Yang, K. Nomoto, M.T. Hasib, O. Muránsky, R. Busch, X. Li, J.J. Kruzic, Fracture and fatigue behaviour of a laser additive manufactured $\mathrm{Zr}$-based bulk metallic glass. Addit. Manuf. 36, 101416 (2020)

29. T.C. Pekin, J. Ding, C. Gammer, B. Ozdol, C. Ophus, M. Asta, R.O. Ritchie, A.M. Minor, Direct measurement of nanostructural change during in situ deformation of a bulk metallic glass. Nat. Commun. 10, 2445 (2019)

30. C.T. Liu, M.F. Chisholm, M.K. Miller, Oxygen impurity and microalloying effect in a Zr-based bulk metallic glass alloy. Intermetallics 10, 1105-1112 (2002)

31. X.H. Lin, W.L. Johnson, W.K. Rhim, Effect of oxygen impurity on crystallization of an undercooled bulk glass forming $\mathrm{Zr}-\mathrm{Ti}-\mathrm{Cu}-$ Ni-Al alloy. Mater. Trans. JIM. 38, 473-477 (1997)

32. J. Eckert, N. Mattern, M. Zinkevitch, M. Seidel, Crystallization behavior and phase formation in $\mathrm{Zr}-\mathrm{Al}-\mathrm{Cu}-\mathrm{Ni}$ metallic glass containing oxygen. Mater. Trans. JIM. 39, 623-632 (1998)

33. S.N. Bogle, L.N. Nittala, R.D. Twesten, P.M. Voyles, J.R. Abelson, Size analysis of nanoscale order in amorphous materials by variable-resolution fluctuation electron microscopy. Ultramicroscopy 110, 1273-1278 (2010) 
34. J.M. Gibson, M.M.J. Treacy, P.M. Voyles, Atom pair persistence in disordered materials from fluctuation microscopy. Ultramicroscopy 83, 169-178 (2000)

35. J. Hwang, P.M. Voyles, Variable resolution fluctuation electron microscopy on $\mathrm{Cu}-\mathrm{Zr}$ metallic glass using a wide range of coherent STEM probe size. Microsc. Microanal. 17, 67-74 (2011)

36. T.E. Gorelik, M.U. Schmidt, U. Kolb, S.J.L. Billinge, Total-scattering pair-distribution function of organic Material from powder electron diffraction data. Microsc. Microanal. 21, 459-471 (2014)

37. P. Juhás, T. Davis, C.L. Farrow, S.J.L. Billinge, PDFgetX3: a rapid and highly automatable program for processing powder diffraction data into total scattering pair distribution functions. J. Appl. Crystallogr. 46, 560-566 (2013)

38. P. Bordeenithikasem, S. Sohn, Z. Liu, J. Schroers, Protocols for multi-step thermoplastic processing of metallic glasses. Scr. Mater. 104, 56-59 (2015)

39. J.P. Best, Z. Evenson, F. Yang, A.-C. Dippel, M. Stolpe, O. Gutowski, M.T. Hasib, X. Li, J.J. Kruzic, Structural periodicity in laser additive manufactured Zr-based bulk metallic glass. Appl. Phys. Lett. 115, 031902 (2019)

40. L.A. Zepeda-Ruiz, A. Stukowski, T. Oppelstrup, N. Bertin, N.R. Barton, R. Freitas, V.V. Bulatov, Atomistic insights into metal hardening. Nat. Mater. 20, 315 (2020)

41. R. Ramachandramoorthy, M. Milan, Z. Lin, S. Trolier-McKinstry, A. Corigliano, H. Espinosa, Design of piezoMEMS for high strain rate nanomechanical experiments. Extrem. Mech. Lett. 20, 14-20 (2018)

42. P.S. Follansbee, C. Frantz, Wave propagation in the split Hopkinson pressure bar. J. Eng. Mater. Technol. 105, 61-66 (1983)

43. D. Jia, K.T. Ramesh, A rigorous assessment of the benefits of miniaturization in the Kolsky bar system. Exp. Mech. 44, 445 (2004)

44. R. Maaß, D. Klaumünzer, J.F. Löffler, Propagation dynamics of individual shear bands during inhomogeneous flow in a Zrbased bulk metallic glass. Acta Mater. 59, 3205-3213 (2011)

45. Z. Han, W.F. Wu, Y. Li, Y.J. Wei, H.J. Gao, An instability index of shear band for plasticity in metallic glasses. Acta Mater. 57, 1367-1372 (2009)

46. B.P. Sahu, A. Dutta, R. Mitra, Mechanism of negative strain rate sensitivity in metallic glass film. J. Alloys Compd. 784, 488-499 (2019)
47. A. Bhattacharyya, G. Singh, K. Eswar Prasad, R. Narasimhan, U. Ramamurty, On the strain rate sensitivity of plastic flow in metallic glasses. Mater. Sci. Eng. A 625, 245-251 (2015)

48. W.H. Jiang, F.X. Liu, F. Jiang, K.Q. Qiu, H. Choo, P.K. Liaw, Strain-rate dependence of hardening and softening in compression of a bulk-metallic glass. J. Mater. Res. 22, 2655-2658 (2007)

49. F.H. Dalla Torre, A. Dubach, M.E. Siegrist, J.F. Löffler, Negative strain rate sensitivity in bulk metallic glass and its similarities with the dynamic strain aging effect during deformation. Appl. Phys. Lett. 89, 91918 (2006)

50. A. Dubach, F.H. Dalla Torre, J.F. Löffler, Deformation kinetics in Zr-based bulk metallic glasses and its dependence on temperature and strain-rate sensitivity. Philos. Mag. Lett. 87, 695-704 (2007)

51. H.M. Chen, J.C. Huang, S.X. Song, T.G. Nieh, J.S.C. Jang, Flow serration and shear-band propagation in bulk metallic glasses. Appl. Phys. Lett. 94, 141914 (2009)

52. T. Mukai, T.G. Nieh, Y. Kawamura, A. Inoue, K. Higashi, Effect of strain rate on compressive behavior of a $\mathrm{Pd}_{40} \mathrm{Ni}_{40} \mathrm{P}_{20}$ bulk metallic glass. Intermetallics 10, 1071-1077 (2002)

53. M.B. Harris, L.S. Watts, E.R. Homer, Competition between shear band nucleation and propagation across rate-dependent flow transitions in a model metallic glass. Acta Mater. 111, 273-282 (2016)

54. Y. Xue, L. Wang, X. Cheng, F. Wang, H. Cheng, H. Zhang, A. Wang, Strain rate dependent plastic mutation in a bulk metallic glass under compression. Mater. Des. 36, 284-288 (2012)

55. S. Hilke, H. Rösner, D. Geissler, A. Gebert, M. Peterlechner, G. Wilde, The influence of deformation on the medium-range order of a Zr-based bulk metallic glass characterized by variable resolution fluctuation electron microscopy. Acta Mater. 171, 275-281 (2019)

56. J.P. Best, J. Ast, B.S. Li, M. Stolpe, R. Busch, X. Li, J. Michler, J.J. Kruzic, Relating fracture toughness to micro-pillar compression response for a laser powder bed additive manufactured bulk metallic glass. Mater. Sci. Eng. A. 770, 138535 (2020)

57. A.E. Lagogianni, C. Liu, K. Martens, K. Samwer, Plastic avalanches in the so-called elastic regime of metallic glasses. Eur. Phys. J. B. 91, 104 (2018)

58. P. Cao, K.A. Dahmen, A. Kushima, W.J. Wright, H.S. Park, M.P. Short, S. Yip, Nanomechanics of slip avalanches in amorphous plasticity. J. Mech. Phys. Solids. 114, 158-171 (2018) 